\title{
Shooting Ahead with Wakefield Acceleration
}

\section{A method for accelerating particles, called wakefield acceleration, has notched up its output energy, bringing it closer to its goal of shrinking the size of accelerator facilities.}

\section{by Florian Grüner*}

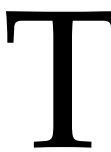
he field of plasma wakefield acceleration is picking up speed. This method, which was first proposed in 1979 [1], creates a collective motion of plasma particles, generating an accelerating field in its wake. The amplitude of this accelerating field is not limited, as it is in conventional acceleration techniques that use radio frequency pulses. The implication is that wakefield acceleration has the potential to work over much smaller lengths, which would allow a reduction in the size (and cost) of accel-
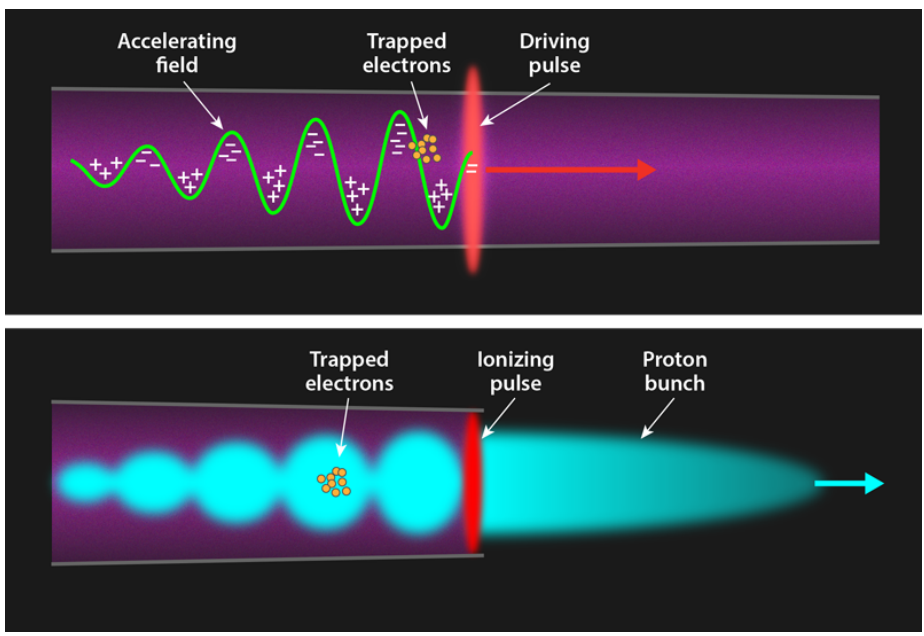

Figure 1: Wakefield acceleration can use different drivers. In the laser-driven case (top), a strong laser pulse is fired into a preformed plasma. The pulse induces charge separation in the plasma, and the electric field from this charge configuration can accelerate trapped electrons. In the proton-driven scenario (bottom), a high-energy proton bunch is sent into a gas. A relatively weak laser pulse ionizes the gas at the mid-point of the bunch, leading to a modulation of the bunch's tail. The resulting microbunches can accelerate electrons injected into the stream. (APS/Alan Stonebraker)

\footnotetext{
* University of Hamburg and Center for Free-Electron Laser Sci-
} ence, Hamburg, Germany erator facilities. There exist different methods for generating wakefields, and now researchers are reporting significant progress for two of these techniques. One method using laser-driven wakefields has generated $8-\mathrm{GeV}$ electrons, a new energy record that doubles the previous record [2]. A different approach, with proton beams as drivers, is not far behind, with recent experiments demonstrating its ability to accelerate electrons up to $2 \mathrm{GeV}$ [3-5]. These are both key achievements, but the goal of having wakefield accelerators will have to wait until researchers gain more control over the output beams.

What is a plasma wakefield? A helpful analogy is a capacitor, where two oppositely charged parallel plates generate an electric field that can accelerate particles from one plate to the other. In a plasma, a similar field can arise when a "driver," such as a laser pulse, separates negatively charged electrons from positively charged ions. This charge separation can remain a stable configuration-the so-called wakefield-if the effective size of the driver is less than the plasma wavelength, which characterizes the length for a coherent response to a charge displacement. Inside the wakefield, the electric field-expressed as a voltage gradient-can reach $1 \mathrm{TV} / \mathrm{m}$. By comparison, conventional accelerators can only reach gradients of $100 \mathrm{MV} / \mathrm{m}$ before they run the risk of being damaged by electrical discharge.

If plasma wakefields can have gradients of $1 \mathrm{TV} / \mathrm{m}$, one might imagine that a "table-top version of CERN" is possible. However, there's a problem. Wakefields are typically driven by a laser pulse, whose speed is significantly reduced inside a plasma. At some point, the electrons will get ahead of the accelerating part of the wakefield, like surfers who outrun the waves they are riding on. There are basically two ways out of this so-called dephasing issue: either one stages several laser-driven plasma accelerator units in succession, or one selects a different driver-a high-energy proton bunch that moves through the plasma at near the speed of light.

If one chooses to go with the laser-driven strategy, each stage needs to give an energy boost of about $10 \mathrm{GeV}$ [6]. In 2004, researchers obtained the first laser-plasma accelerated electrons with a "nonthermal" energy spectrum peaking at around $100 \mathrm{MeV}$ [7]. Two years later, the peak energy 
reached $1 \mathrm{GeV}$ [8]. Now, Anthony Gonsalves from Lawrence Berkeley National Laboratory, California, and colleagues have managed to accelerate electrons to $8 \mathrm{GeV}$ with lasers [2], which makes this an important milestone in reaching the needed $10-\mathrm{GeV}$ energy per stage.

So, why did it take more than 10 years to go from $1 \mathrm{GeV}$ to $8 \mathrm{GeV}$ ? One reason is the complexity of these experiments. The first-generation laser-plasma experiments operated with just one laser, which both created the plasma (through gas ionization) and generated the wakefield. The next generation relegated the ionization step to a discharge capillary, which is a gas container that is typically made out of glass, with an inner diameter smaller than that of a human hair. When a strong voltage is applied to the capillary, the discharge ionizes the gas, creating a plasma whose density is lowest along the tube axis, as the walls are cooling faster than the center (Fig. 1, top). This transverse density gradient helps guide the laser pulse over sufficiently long acceleration lengths of around $10 \mathrm{~cm}$. Gonsalves et al. have improved on this design by using a second laser system in addition to the discharge capillary. This second laser acts as a "laser heater," increasing the transverse plasma density gradient even further-a prerequisite for reaching the $8-\mathrm{GeV}$ level.

As already mentioned, the laser-driven method will require coordinating multiple stages, a feat that remains to be tackled. The alternative approach is to use a single high-energy proton bunch as a driver. The difficulty here is that the proton bunch is initially much longer than the plasma wavelength, which would normally mean that no stable wakefield can arise. Fortunately, nature provides a solution: proton-plasma interactions cause the proton bunch to spontaneously form a density modulation consisting of "microbunches" whose spacing is roughly that of the plasma wavelength. Stable wakefields arise in between the microbunches (Fig. 1, bottom). To control this so-called selfmodulation, researchers can use a laser-induced ionization front that acts as a "seed" for the breakup of the proton bunch.

Early in 2018, the Advanced Wakefield Experiment (AWAKE) made the first successful demonstration of protondriven acceleration, using $400-\mathrm{GeV}$ proton bunches from the Super Proton Synchrotron at CERN [3]. The researchers showed that they could achieve a 2-GeV energy boost for electrons that were injected into the wake of these proton bunches. In a pair of follow-up studies published earlier this month, the AWAKE Collaboration verified that the roughly 10 -cm-long proton bunches break up into microbunches that are about $2 \mathrm{~mm}$ long, as predicted for seeded selfmodulation [4, 5]. The acceleration occurs over $10 \mathrm{~m}$, which is roughly 100 times longer than the acceleration length in the laser-driven experiment. The longer distance is due to the fact that the proton-bunch experiment requires a lower plasma density and hence a weaker electric field gradient. However, the proton-based scheme may prove better suited than the laser-driven case for accelerating positrons. The positrons could be simply injected into the wake of the proton beam in the same way as electrons are.

The AWAKE Collaboration and Gonsalves et al. have both achieved landmark results, but much work remains. The CERN approach is aimed at studying the possibilities of a novel high-energy electron-positron collider, while the laserbased method targets not only collider applications but also the driving of hard x-ray free-electron lasers, which are used, for instance, to determine protein structures. It is really difficult to judge which approach is closer to its goal, simply because both methods are still busy demonstrating acceleration. To go to the next step of creating a user facility, wakefield acceleration requires high beam quality and stability, which neither technique has achieved so far.

We often ask: when was the starting point of a new field? Did lunar exploration, for example, begin with Jules Verne's 1865 novel about a voyage to the moon, with the first human in space in 1961, or with the moon landing in 1969? For wakefield acceleration, there are several potential starting points. In my view, the "human-in-space" moment for plasma wakefield acceleration took place in 2004 when researchers managed to achieve $100-\mathrm{MeV}$ energies with quasimonochromatic energy spectra. It is still not clear when the "moon landing" will happen in the form of a wakefield-accelerator user facility. The key to reaching this goal will be to develop an unprecedented level of control over all relevant parameters. This means introducing entirely new "knobs" for things like the final energy and energy spread of the accelerated particles.

This research is published in Physical Review Letters and Nature.

\section{REFERENCES}

[1] T. Tajima and J. M. Dawson, "Laser electron accelerator," Phys. Rev. Lett. 43, 267 (1979).

[2] A. J. Golsalves et al., "Petawatt laser guiding and electron beam acceleration to $8 \mathrm{GeV}$ in a laser-heated capillary discharge waveguide," Phys. Rev. Let 122, 084801 (2019).

[3] E. Adli et al. (AWAKE Collaboration), "Acceleration of electrons in the plasma wakefield of a proton bunch," Nature 561, 363 (2018).

[4] M. Turner et al. (AWAKE Collaboration), "Experimental observation of plasma wakefield growth driven by the seeded selfmodulation of a proton bunch," Phys. Rev. Lett. 122, 054801 (2019).

[5] E. Adli et al. (AWAKE Collaboration), "Experimental observation of proton bunch modulation in a plasma at varying plasma densities," Phys. Rev. Lett. 122, 054802 (2019).

[6] C. B. Schroeder, E. Esarey, C. G. R. Geddes, C. Benedetti, W. P. Leemans et al., "Physics considerations for laser-plasma linear colliders," Phys. Rev. ST Accel. Beams 13, 101301 (2010).

[7] C. G. R. Geddes, Cs. Toth, J. van Tilborg, E. Esarey, C. B. Schroeder, D. Bruhwiler, C. Nieter, J. Cary, and W. P. Leemans, 
"High-quality electron beams from a laser wakefield accelerator using plasma-channel guiding," Nature 431, 538 (2004); S. P. D. Mangles et al., "Monoenergetic beams of relativistic electrons from intense laser-plasma interactions," Nature 431, 535 (2004); J. Faure, Y. Glinec, A. Pukhov, S. Kiselev, S. Gordienko, E. Lefebvre, J.-P. Rousseau, F. Burgy, and V. Malka, "A laser-plasma accelerator producing monoenergetic electron beams," Nature 431, 541 (2004).

[8] W. P. Leemans, B. Nagler, A. J. Gonsalves, Cs. Tóth, K. Nakamura, C. G. R. Geddes, E. Esarey, C. B. Schroeder, and S. M. Hooker, "GeV electron beams from a centimetre-scale accelerator," Nat. Phys. 2, 696 (2006).

10.1103/Physics.12.19 